\title{
Design principles for the ultimate gas deliverable capacity material: nonporous to porous deformations without volume change
}

\author{
Matthew Witman, ${ }^{*} \dagger$ Sanliang Ling, ${ }^{\ddagger}$ Vitalie Stavila, ${ }^{\dagger}$ Pavithra Wijeratne, ${ }^{\dagger}$ \\ Hiroyasu Furukawa, ${ }^{\mathbb{9}, \S}$ and Mark D. Allendorf*, ${ }^{*}$ \\ $\dagger$ Sandia National Laboratories, Livermore, California 94551, USA \\ $\ddagger$ Advanced Materials Research Group, Faculty of Engineering, University of Nottingham, \\ University Park, Nottingham, UK \\ IDepartment of Chemistry, University of California, Berkeley, CA 94720, USA \\ $\S$ Materials Sciences Division, Lawrence Berkeley National Laboratory, Berkeley,CA 94720, \\ $U S A$ \\ E-mail: mwitman@sandia.gov; mdallen@sandia.gov
}

\section{Abstract}

Understanding the fundamental limits of gas deliverable capacity in porous materials is of critical importance as it informs whether technical targets (e.g., for on-board vehicular storage) are feasible. High-throughput screening studies of rigid materials, for example, have shown they are not able to achieve the original ARPA-E methane storage targets, yet an interesting question remains: what is the upper limit of deliverable capacity in flexible materials? In this work we develop a statistical adsorption model that specifically probes the limit of deliverable capacity in intrinsically flexible materials. The resulting adsorption thermodynamics indicate that a perfectly designed, intrinsically flexible nanoporous material could achieve higher methane deliverable capacity than the best benchmark systems known to date with little to no total volume change. Density functional theory and grand canonical Monte Carlo simulations identify a known metal-organic framework (MOF) that validates key features of the model. Therefore, this work (1) motivates a continued, extensive effort to rationally design a porous mate-
\end{abstract}

rial analogous to the adsorption model and (2) calls for continued discovery of additional high deliverable capacity materials that remain hidden from rigid structure screening studies due to nominal non-porosity.

\section{Introduction}

The economic viability of gas storage technologies for cleaner vehicle transportation hinges on materials with sufficiently large deliverable capacity, defined as the difference in gas loading between the charging and discharging pressures. ${ }^{1-4}$ There have therefore been concerted efforts to understand the physical limit of deliverable capacity in porous materials, especially within the applications of hydrogen and methane storage. ${ }^{5-8}$ We will provide evidence that, through a carefully designed analytical model and corroborating calculations on known materials, the deliverable capacities of today's benchmark materials still have not reached a fundamental limit if intrinsic flexibility can be deftly exploited. While this work uses methane storage as a case study, the lessons learned are general and could be applied to other gas stor- 
age applications. ${ }^{9}$

Typical computational exploration of gas deliverable capacity in porous materials consists of screening large databases of known and hypothetical materials using the rigid structure approximation. ${ }^{5-8}$ For example, these studies have convincingly revealed that the original Advanced Research Project Agency-Energy (ARPA-E) methane deliverable capacity (DC) targets of $315 \mathrm{v}(\mathrm{STP}) / \mathrm{v}$ delivered at ambient temperature between 5.8 and 65 bar are not achievable using the materials which have been screened under the rigid framework approximation. ${ }^{7}$ One fundamental problem is that strong methane interactions are needed to achieve high capacities at the charging pressure, but this results in a capacity that is also too high at the discharging pressure, thereby reducing the deliverable capacity to $\sim 70 \%$ or less of the high pressure adsorption. This realization has also been confirmed by a host of modeling studies that elucidate the deliverable capacity upper bound in rigid materials. ${ }^{10,11}$ Temperature swing operation can address this decrease in deliverable capacity, ${ }^{12}$ but at the cost of additional weight for the necessary heating/cooling systems. Extrinsically flexible materials on the other hand, ${ }^{13-15}$ which deliver gas via an S-shaped isotherm (e.g., IUPAC Type $\mathrm{V}$ isotherm) due to a large volume expansion from a nonporous to a porous state, have been investigated thoroughly both computationally and experimentally in an attempt to overcome this physical limit. ${ }^{16-19}$ However, it is unclear how well these large volume change materials can be practically utilized from a systems design standpoint, as they must remain functional after many cycles despite shear displacive phase transformations. ${ }^{20,21}$ Current technology development efforts appear to forsake the enhanced deliverable capacity of extrinsically flexible materials, and instead focus on constant volume, rigid adsorbents that can be densified with robust mechanical stability. ${ }^{22}$ In the search for materials that improve upon state-of-the-art methane $D C$ targets, here we diverge from the approaches above.

First, we design an adsorption model of an intrinsically flexible material ${ }^{23-26}$ (exhibits a pore volume change but no significant unit cell volume change) that offers the same favorable adsorption properties of an extrinsically flexible material. We describe a flexible slit pore model that provides a physical basis for designing high deliverable capacity materials, whereby an "ideal" material could translate nearly all of the adsorption capacity to deliverable capacity (see Figure 1 for an example). We specifically design the minimum energy configuration of the pore to be too small to accommodate adsorbates, and the fully porous state is accessible at higher energy, which can be stabilized by the presence of adsorbates. ${ }^{27}$ The deliverable capacity can therefore be enhanced due to the reduced uptake at low pressures (while maintaining an equally high uptake at high pressures) to the point that it competes with or even exceeds today's benchmark materials. ${ }^{19,22,28}$

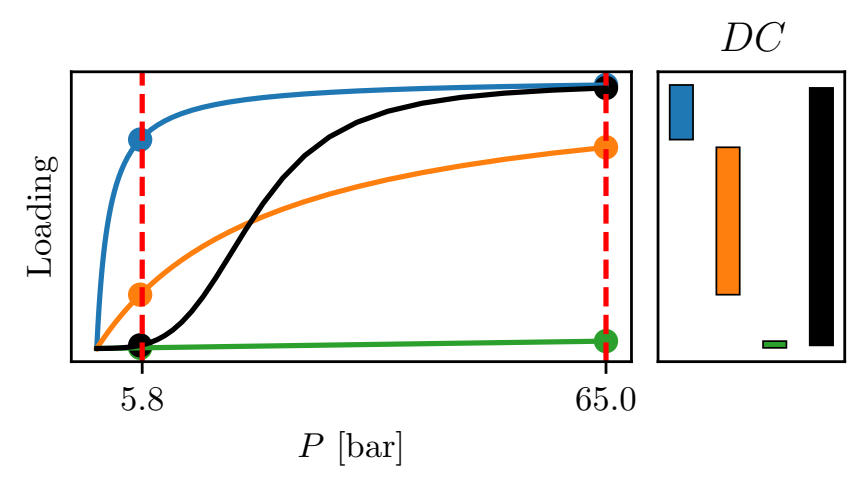

Figure 1: Example rigid material isotherms where adsorption is either too energetically favorable (blue), optimized (orange), or too unfavorable (green) for maximizing $D C$. Flexible systems (black) have the potential to maximize $D C$ by suppressing adsorption at low pressures without sacrificing high capacity at high pressures.

Second, rather than computationally screening hundreds of thousands of materials within the rigid structure approximation, we perform a set of detailed density functional theory (DFT) and grand canonical Monte Carlo (GCMC) calculations on a family of intrinsically flexible materials, known as $\mathrm{M}(\mathrm{NDC})(\mathrm{M}=\{\mathrm{Ca}, \mathrm{Sr}\}$, $\mathrm{NDC}=1,4$-naphthalenedicarboxylate). ${ }^{29}$ DFT calculations validate some of the key geometric/energetic features of the flexible slit pore model that impart a high deliverable capacity. 
We initially selected this material because DFT relaxation of the desolvated structure resulted in a large pore volume change but very little unit cell volume change. Despite their "nominal non-porosity" in the minimum energy state (i.e. the pore size of the minimum energy configuration of the framework is too small to accommodate methane), these materials are subject to significant changes in the pore size/shape with minimal change to the unit cell volume. The transition from nonporous to porous becomes thermodynamically favorable if, at high chemical potentials, adsorbate-framework interactions reduce the free energy of a porous state that is otherwise higher in free energy than the nonporous state in the absence of adsorbates at low temperature. ${ }^{26,30}$ In similar systems, cooperative adsorption due to ring rotations in constant volume materials has been modeled and observed (although the minimum energy state was still porous and such studies were not intended to probe deliverable capacity limits). ${ }^{31,32}$ It should be noted that such materials could only ever be computationally identified with a fully flexible treatment coupling framework dynamics and adsorption; ${ }^{17,33-36}$ hence, they will have remained hidden from almost any high-throughput adsorbent screening study to date. ${ }^{37,38}$ Finally, we show that the isoreticular tunability ${ }^{39,40}$ of metal-organic frameworks (MOFs) can be used to design an improved version of the $\mathrm{M}(\mathrm{NDC})$ adsorbent. Ultimately, this work outlines a path forward to discover deliverable capacity materials through concrete design strategies motivated by the models and simulations developed herein.

\section{Flexible Slit Pore Model}

We derive a numerically-solvable adsorption model that accounts for intrinsic flexibility via a rotating and vibrating slit pore. This allows the adsorption properties to be quickly and systematically solved over the model parameter space and provides insights into deliverable capacity limits in intrinsically flexible materials.

\section{Slit pore geometry}

Figure 2 visualizes our slit pore adsorption model where the centerlines of the pore walls are separated by a distance $L$. The tilt of the pore, $\theta$, is the angle between the centerline and the normal to the $i, k$ crystallographic plane, where the $k$ direction is out of the page in Figure 2 and explicitly visualized in Figure 4. $x$, the adsorbate's degree of freedom, is the magnitude of its displacement vector from the centerline of the left hand wall in Figure 2, parallel with $i$. The slit pore is large enough to ignore edge effects such that the particle's adsorption energy will be independent of its coordinates in the $j$ and $k$ (into the page) directions. $x_{\perp}$ is the magnitude of the displacement vector normal to the centerline of the wall. Therefore the minimum distance to the first wall is given by $x_{\perp, 1}(x, \theta)=x \cos \theta$ and the minimum distance to the opposite wall by $x_{\perp, 2}(x, \theta, L)=(L-x) \cos \theta$. The largest included sphere that can fit without overlapping the pore wall is

$$
D_{i}(\theta, L)=L \cos \theta-\sigma_{F},
$$

where $\sigma_{F}$ is the Lennard-Jones diameter of the pore wall.

This model will enable prediction of adsorption thermodynamics with numerical integration rather than Monte Carlo simulations, and despite its simplicity, a close analog of this rotating slit pore can be found within a known MOF (visualized in Figure 2 and discussed later). At $\theta=0, L$ can be chosen such that $D_{i}$ is slightly larger than the adsorbate and maximizes the binding strength. Then, if $L$ remains constant, increasing $\theta$ decreases $D_{i}$ until the slit pore becomes nonporous. If the nonporous state is energetically preferred but the porous state can be stabilized by the presence of adsorbates, a significant pore volume change can be achieved without a significant cell volume change. This has the potential to provide enhanced $D C$, which we quantitatively explore with the model. 

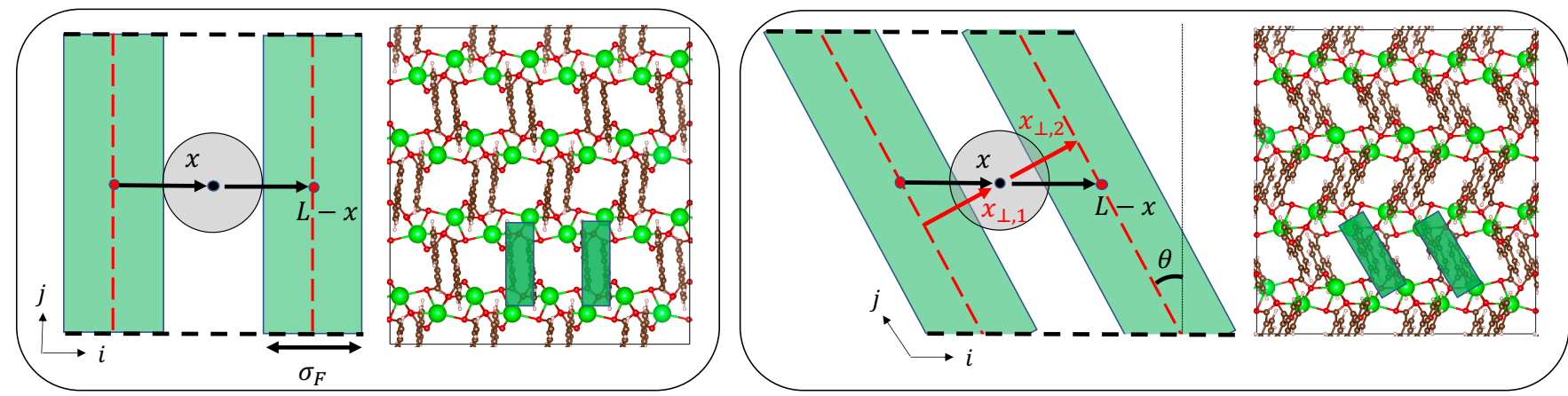

Figure 2: The relevant geometry parameters for computing the energetics between an adsorbate (gray circle) and the slit pore walls (green rectangles). Red dashed lines represent the center line of each wall, red dots represent the rotation center for each wall, and the black dots represents the adsorbate center. We also show an example of a known MOF, Sr(NDC), we discovered to have rotating slit pores when DFT relaxation of the desolvated structure resulted in a large pore volume change but very little unit cell volume change.

\section{Framework-guest potential}

Lennard-Jones interactions describe frameworkguest interactions, and the methane guest parameters $\epsilon_{G}=148 \mathrm{~K}$ and $\sigma_{G}=3.73 \AA$ are taken from TraPPE. ${ }^{41}$ The framework consists of carbon atoms with $\epsilon_{F}=52.8 \mathrm{~K}$ and $\sigma_{F}=3.43 \AA$ taken from the Universal Force Field. ${ }^{42}$ Each pore wall is treated as a plane of uniformly smeared Lennard-Jones spheres with planar density, $d_{s}$, equal to graphene. The potential energy between methane and one of the smeared carbon walls, $E_{s}$, can then be derived as a function of $x_{\perp},{ }^{43}$

$$
E_{s}\left(x_{\perp}\right)=2 \pi \epsilon \sigma^{2} d_{s}\left[\frac{2}{5}\left(\frac{\sigma}{x_{\perp}}\right)^{10}-\left(\frac{\sigma}{x_{\perp}}\right)^{4}\right] .
$$

$\epsilon$ and $\sigma$ are determined by Lorentz-Berthelot combining rules, $\epsilon=\sqrt{\epsilon_{G} \epsilon_{F}}$ and $\sigma=\left(\sigma_{G}+\right.$ $\left.\sigma_{F}\right) / 2$. This leads to the final adsorption energy

$E_{F G}(x, \theta, L)=E_{s}\left(x_{\perp, 1}(x, \theta)\right)+E_{s}\left(x_{\perp, 2}(x, \theta, L)\right)$,

where $E_{s}\left(x_{\perp, 1}(x, \theta)\right)$ yields the interaction energy with the first wall and $E_{s}\left(x_{\perp, 2}(x, \theta, L)\right.$ yields the interaction energy with the second wall.

\section{Framework potential}

We consider three different forms of the framework potential energy, $E_{F}$, corresponding to the different types of flexibility given by eqs. (4) to $(6)$.

$$
\begin{aligned}
E_{F}^{S}(\theta, L) & = \begin{cases}0 & \theta=\theta_{\text {eq }}, L=L_{e q} \\
\infty & \text { otherwise. }\end{cases} \\
E_{F}^{R}(\theta, L) & = \begin{cases}\frac{V_{\max }}{b^{4}}\left(\left(\theta-\frac{a}{2}\right)^{2}-b^{2}\right)^{2}-c \theta & L=L_{e q} \\
\infty & \text { otherwise. }\end{cases} \\
E_{F}^{R+V}(\theta, L) & =\frac{V_{\max }}{b^{4}}\left(\left(\theta-\frac{a}{2}\right)^{2}-b^{2}\right)^{2}-c \theta+k\left(L-L_{e q}\right)^{2}
\end{aligned}
$$


1. In the static (S) slit pore of eq. (4), $\theta$ and $L$ are fixed at $\theta_{e q}$ and $L_{e q}$ and do not fluctuate.

2. In the rotating $(\mathrm{R})$ slit pore of eq. (5), $\theta$ fluctuates in a skewed double well potential, the shape of which is controlled by $V_{\text {max }}, a, b$, and $c . \quad L$ is fixed at $L_{e q}$ and does not a fluctuate.

3. In the rotating and vibrating $(\mathrm{R}+\mathrm{V})$ slit pore of eq. (6), $L$ fluctuates according to a harmonic spring potential with spring constant $k$.

Choosing $V_{\text {max }}=20 \mathrm{~kJ} / \mathrm{mol}, a=\pi / 6, b=$ $\pi / 12, c=50 \mathrm{~kJ} / \mathrm{mol}$ creates an $E_{F}$ profile similar to the MIL-53 breathing free energy profile, ${ }^{44}$ and the effect of parameter variation on the adsorption performance is examined further in S1.1. The global minimum of $E_{F}^{R}$ with these parameters occurs at $\theta^{\prime}=31.1^{\circ}$ and corresponds to an asymmetric double well, ${ }^{45}$ although different parameter sets (see Figure 3) can yield asymmetric single well potentials and still produce similarly favorable deliverable capacity enhancement (see S1.1 for more details). Interesting future work could elucidate just how much of the overall parameter space leads to an equally enhanced deliverable capacity.

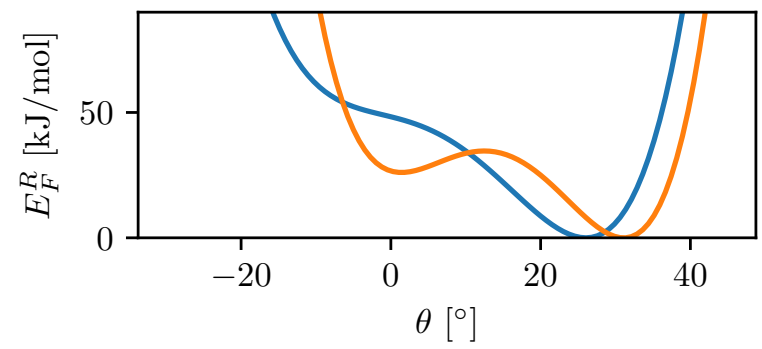

Figure 3: Visualization of (5) for two parameter sets that yield either asymmetric single or double well potentials, adjusted by a constant shift such that the global minimum occurs at $E_{F}^{R}=0$. The orange curve shows the parameter set used in the main article.

\section{Adsorption volume discretization}

Next we discretize the adsorption space into lattice sites and disallow multi-site occupancy to model repulsive particle overlaps. Note that site discretization is similarly used to derive the Langmuir isotherm from statistical mechanics. ${ }^{10}$ Specifically, the adsorption space is discretized into $M$ channels in the $j, k$ plane as shown in Figure 4. Each channel site has

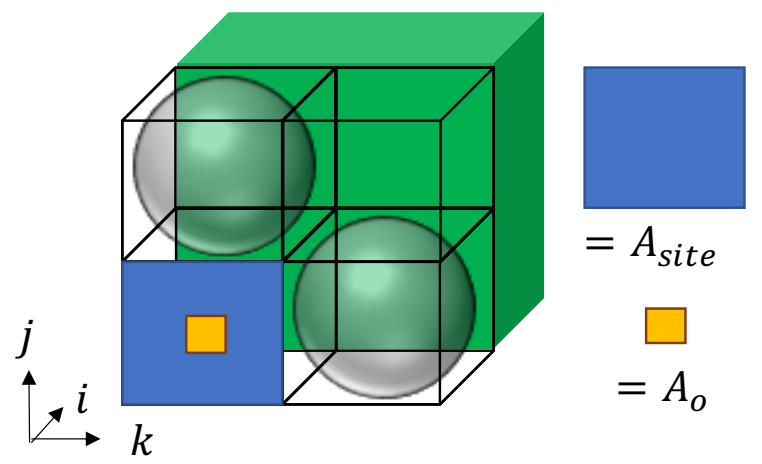

Figure 4: Discretization of the adsorption domain from Figure 2 into $M=4$ lattice channels in the $(j, k)$ plane with $N=2$ adsorbates, each with total and free areas, $A_{\text {site }}$ and $A_{o}$, respectively.

a cross-sectional area of $A_{\text {site }}=\left(2^{1 / 6} \sigma_{G}\right)^{2}=$ $(4.19 \AA)^{2}$ with a volume of $V_{\text {site }}=A_{\text {site }} L$. The calculation of the free cross-sectional area, $A_{o}=$ $1.1 \AA^{2}$, is outlined in S1.4 and its accuracy is ensured later (see Figure 7). In short, this quantity refers to the area in which the particle can translate without overlapping a neighboring adsorption site. While explicitly preventing particle overlaps (i.e., modeling hardsphere repulsion), this discretization does not account for favorable guest-guest interactions. However, due to the two-dimensional nature of the pore space, the adsorbates in the slit pore have a limited number of close neighbors relative to larger three-dimensional pore spaces. Each methane-methane interaction could contribute at most $\epsilon=-1.2 \mathrm{~kJ} / \mathrm{mol}$ (minimum in the methane-methane interaction potential), making it a small contribution relative to the interaction between methane and the framework in this particular slit pore system. Guest-guest interactions could of course be accounted for explicitly, but then we would rely on MC simulations to solve the model due to the complexity of the resulting partition function. Alternatively, future work could explore mean field 
approximations to incorporate guest-guest interactions without resorting to MC simulations. Therefore, the single site partition function for an adsorbed methane molecule in one of the site channels becomes

$$
q(\theta, L)=\frac{A_{o}}{\Lambda^{3}} \int d x \exp \left[-\beta E_{F G}(x, \theta, L)\right],
$$

where $\Lambda$ is the thermal de Broglie wavelength.

We note that as $L$ becomes sufficiently large relative to the size of the adsorbate in question, the assumptions of this discretization breakdown. For sufficiently large $L$, more adsorption sites will be created (envision, for example, a two layer hexagonal close packing of methane in the slit pore). However, we are not interested in these larger $L$ because the purpose of the slit pore is to remain nonporous in its rotated state. Requiring $\theta \rightarrow 90^{\circ}$ to maintain nonporosity of the rotated state could complicate their embedding in real materials and even introduce porosity in an unanticipated dimension (an example of introducing undesired porosity is shown in S2.3). The $L$ range over which this discretization strategy is valid is discussed further in the results.

\section{Canonical partition function}

The canonical partition functions corresponding to each type of flexible slit pore are expressed as a product of single site partition functions with integration over the framework degrees of freedom, $\theta$ and $L$, in eqs. (8) to (10).

$$
\begin{aligned}
& Q^{S}\left(N, M, \beta ; \theta_{e q}, L_{e q}\right)=\left(\begin{array}{c}
M \\
N
\end{array}\right) q\left(\theta_{e q}, L_{e q}\right)^{N} \\
& Q^{R}\left(N, M, \beta ; L_{e q}\right)=\left(\begin{array}{c}
M \\
N
\end{array}\right) \int d \theta \exp \left[-\beta E_{F}^{R}\left(\theta, L_{e q}\right)\right] q\left(\theta, L_{e q}\right)^{N} \\
& Q^{R+V}(N, M, \beta)=\left(\begin{array}{c}
M \\
N
\end{array}\right) \int d \theta \int d L \exp \left[-\beta E_{F}^{R+V}(\theta, L)\right] q(\theta, L)^{N}
\end{aligned}
$$

Here $\beta=\left(k_{B} T\right)^{-1}$ where $k_{B}$ is Boltzmann's constant and $T$ is temperature, and $\left(\begin{array}{c}M \\ N\end{array}\right)$ accounts for the indistinguishability of particles and enforces single occupancy sites. These one, two, or three dimensional integrals can be evaluated using standard numerical integration libraries contained in scipy or Mathematica (see S1.5 for code availability).

We note that, due to the fluctuating $L$ in the $\mathrm{R}+\mathrm{V}$ model, the natural ensemble of a single slit pore is actually the osmotic ensemble rather than the grand ensemble. For practical purposes however, we are interested in rotating slit pores that are embedded within a rigid support. So while an individual slit pore may vibrate and fluctuate in $L$, the overall crystallite volume remains constant (as is visualized in Figure 2 and later demonstrated via DFT calculations). In such a case it is more realistic that the free en- ergy of adsorption in a $\mathrm{R}+\mathrm{V}$ slit pore is coupled only to the reservoir through the chemical potential and not the mechanical pressure. The analytical tractability of the model would be lost by investigating adsorption in multiple, coupled $\mathrm{R}+\mathrm{V}$ slit pores in the grand ensemble but portends interesting future work that accounts for volume changes in individual $\mathrm{R}+\mathrm{V}$ slit pores.

\section{Isotherms, adsorption enthalpy, and deliverable capacity}

Solving the canonical partition function permits calculation of the grand partition function. Then the adsorption isotherm, or the expectation number of particles, $\langle N\rangle$, as a function of 
$\mu$, the chemical potential, can be computed as

$$
\langle N\rangle(\mu, M, \beta)=\frac{\sum_{N=0}^{M} N \exp (\beta \mu N) Q(N, M, \beta)}{\sum_{N=0}^{M} \exp (\beta \mu N) Q(N, M, \beta)} .
$$

A material's $D C$ is the difference in adsorbate loading between the adsorption pressure, $P_{a d s}$, and the desorption pressure, $P_{d e s}<P_{a d s}$,

$$
D C=\langle N\rangle\left(P_{a d s}, M, \beta\right)-\langle N\rangle\left(P_{d e s}, M, \beta\right) .
$$

We relate $\mu$ to $P$ through the ideal gas law, ${ }^{46}$ a good approximation at ambient temperatures for light gases such as methane. For the DOE storage targets, $P_{\text {des }}=5.8$ bar and $P_{a d s}=65$ bar (see Figure 1). Finally, we can compute the differential enthalpy of adsorption via the Clausius-Clapeyron formalism from isotherms obtained at multiple temperatures,

$$
\Delta \bar{h}=R\left(\frac{\partial \ln \left(P / P_{0}\right)}{\partial(1 / T)}\right)_{N}+R T,
$$

where $R$ is the gas constant and $P_{0}=1$ bar is the ideal gas reference state pressure. $\Delta \bar{h}$ gives the amount of heat that must be added/removed from the system to maintain constant temperature when transferring a molecule from the gas phase to the adsorbent, or vice versa, at a given loading. ${ }^{47,48}$

\section{DFT flexibility calculations}

Rigorous atomistic simulations of adsorption in flexible nanoporous materials are complicated endeavors, not only because they often require enhanced sampling techniques, ${ }^{34,36}$ but also because the predictions can be very sensitive to small fluctuations in structural dynamics. ${ }^{37}$ In other words, quantitative isotherm predictions require an extremely accurate description of the potential energy surface and computationally intensive simulations. Each challenges either classical force fields (do not universally transfer with high accuracy across the diverse MOF chemistry/structure space) or ab initio molecular dynamics (are very expensive). We therefore turn to a simple DFT screening procedure to gauge if a material with a rotating slit pore has potential for an intrinsic nonporous to porous transitions.

We calculate two contributions to the potential energy in a nonporous to porous transition, the framework deformation energy and the adsorption energy, by considering the empty framework, F, or the framework with guest adsorbates, $\mathrm{F} \cdot \mathrm{G}$. The system of interest $X$ is labeled by $\left.X\right|_{Y} ^{Z}$, where $Y$ is the system from which the geometry of $X$ was taken. $Z \in$ $\{$ DFT, Exp. $\}$ denotes whether the geometry of $Y$ was determined by a DFT relaxation or taken from experimental measurements. So, F| $\left.\right|_{F \cdot G} ^{D F T}$ denotes that we perform a DFT optimization of the framework with adsorbates present but then keep only the framework. $\left.F\right|_{F \cdot G} ^{\text {Exp. }}$ denotes we took the experimental CIF file and removed the adsorbates/solvent that were present, and $\left.\mathrm{F}\right|_{\mathrm{F}} ^{\mathrm{DFT}}$ indicates we have DFT optimized the empty framework. The DFT computed energy of these configurations is denoted $E\left(\left.X\right|_{Y} ^{Z}\right)$, and so the total energy required to deform the framework to accommodate a given set of adsorbates, $\Delta E_{\text {def }}$, the total binding energy of those adsorbates, $\Delta E_{b}$, and the net energy change $\Delta E_{n e t}$ are expressed as

$$
\begin{aligned}
\Delta E_{\text {def }} & =E\left(\left.\mathrm{~F}\right|_{\mathrm{F} \cdot \mathrm{G}} ^{\mathrm{DFT}}\right)-E\left(\left.\mathrm{~F}\right|_{\mathrm{F}} ^{\mathrm{DFT}}\right) \\
\Delta E_{b} & =E\left(\left.\mathrm{~F} \cdot \mathrm{G}\right|_{\mathrm{F} \cdot \mathrm{G}} ^{\mathrm{DFT}}\right)-E\left(\left.\mathrm{~F}\right|_{\mathrm{F} \cdot \mathrm{G}} ^{\mathrm{DFT}}\right) \\
\Delta E_{n e t} & =\Delta E_{d e f}+\Delta E_{b} .
\end{aligned}
$$

These can be normalized by the $N$ number of adsorbates in the $\mathrm{F} \cdot \mathrm{G}$ configuration: $\Delta \bar{E}_{\text {def }}=$ $\Delta E_{\text {def }} / N, \Delta \bar{E}_{b}=\Delta E_{b} / N$, and $\Delta \bar{E}_{n e t}=$ $\Delta E_{\text {net }} / N$. Details on the DFT settings used to calculate the energies and perform the structural optimizations are provided in S2.1. ${ }^{49-59}$

\section{Results}

First, we predict the adsorption properties of the flexible slit pore model. Mapping the model's $D C$ to a volumetric basis then provides useful insights into the maximum achievable $D C$ in intrinsically flexible materials and whether such a material could exceed the performance of the current benchmark systems. 
Next, we utilize DFT to probe the adsorption energy landscape of a known material that exhibits some of the advantages featured by the adsorption model. Finally, we perform an isoreticular design exercise to improve its potential for methane storage.

\section{Adsorption in flexible slit pores}

The main limitation of rigid materials ${ }^{6,7,10}$ is briefly summarized by the static pore isotherms in Figure 5, where the deliverable capacity is calculated as a function of pore wall separation distance (at fixed $\theta_{e q}=0$ ). Two maximums in the deliverable capacity occur when the methane binding energy is neither too strong nor too weak. In the intermediary local minimum, the adsorption is maximized at 65 bar due to highly favorable methane interactions, but the $D C$ suffers because too much methane is also adsorbed at 5.8 bar. Such behavior is also observed in the deliverable capacity simulations of Ref. 60 where inter-layer spacing of 2D COF structures was varied computationally. This plot summarizes the fundamental limitation of gas storage in rigid materials, and, even for the record methane storage in rigid materials, the deliverable capacity is $\sim 30 \%$ less than the adsorption capacity at 65 bar. $^{22}$

In contrast, the $\mathrm{R}$ and $\mathrm{R}+\mathrm{V}$ slit pores avoid this trade-off and allow nearly $100 \%$ of the material's adsorption capacity to be utilized as deliverable capacity. When $L_{e q}$ is too small, even a pore tilt of $\theta=0$ yields a nonporous material, and so the uptake and $D C$ are negligible. When $L_{e q}$ is too large, we recover Langmuirian adsorption because the framework is porous for any value of $\theta$. The "sweet-spot" for achieving an optimal $D C$ is a mere $\sim 0.5 \AA$ window in $L_{e q}$. At these separation distances, the framework adopts a nonporous state at $\theta^{\prime}$ in the absence of any adsorbates, while the porous state $(\theta \sim 0)$ is stabilized at sufficiently high pressures by the presence of adsorbates. This leads to an inflection in the isotherm, whereby adsorption is suppressed at low pressures and deliverable capacity is increased. A critical note is that this S-shaped isotherm behavior can only be achieved in this system due to "coop- erative adsorption", i.e. if $M>1$. In S1.2 we elaborate more on this topic and demonstrate how $D C$ varies with $M$, i.e. the number of adsorption sites that exist in the open slit pore. In other statistical mechanics models of flexible adsorbents, this cooperativity manifests through different mechanisms but still causes inflections in the isotherm. ${ }^{27,31}$ Framework vibrations at finite temperatures can have a nonnegligible impact on the pore size distribution and therefore a porous material's separation capabilities, ${ }^{37}$ so it is important to note that the optimal deliverable capacity of the $\mathrm{R}+\mathrm{V}$ pore is only marginally affected by the inclusion of vibrations. Namely, the maximal $D C$ is slightly reduced and occurs at slightly lower $L_{e q}$, which is needed to ensure that the nonporous state remains nonporous despite $L>L_{e q}$ fluctuations at low chemical potential, but not so low that $\langle L\rangle$ still cannot reach a porous structure at high chemical potential. As the spring constant is reduced, the optimal $L_{e q}$ value continues to shift lower (see S1.3). For $L_{e q}>9 \AA$, our site discretization approximations break down (see later results in Figure 7); regardless, flexible slit pore isotherms approaching this regime have already recovered their Langmuirian behavior because even the rotated state is porous and can't suppress the low pressure uptake necessary to drastically enhance $D C$.

\section{Intrinsic heat management}

The flexible slit pore model demonstrates intrinsic heat management in the same way as extrinsically flexible materials. ${ }^{19}$ The positive energy penalty required to deform the adsorbent offsets some of the energy released during an adsorption event, leading to a reduction in the differential enthalpy of adsorption. Figure 6 shows $\Delta \bar{h}$ computed for the $\mathrm{R}$ and $\mathrm{S}$ slit pores. In this plot, $\theta_{e q}=0$ for $\mathrm{S}$. For both $\mathrm{S}$ and $\mathrm{R}$, the $L_{e q}$ that maximizes deliverable capacity is used (6.7 and $7.2 \AA$ respectively from Figure 5). Since the static slit pore corresponds to Langmuirian adsorption, $\Delta \bar{h}$ is constant as a function of $N$. While the rotating slit pore still shares some Langmuirian assumptions, the underlying $E_{F}$ profile results 


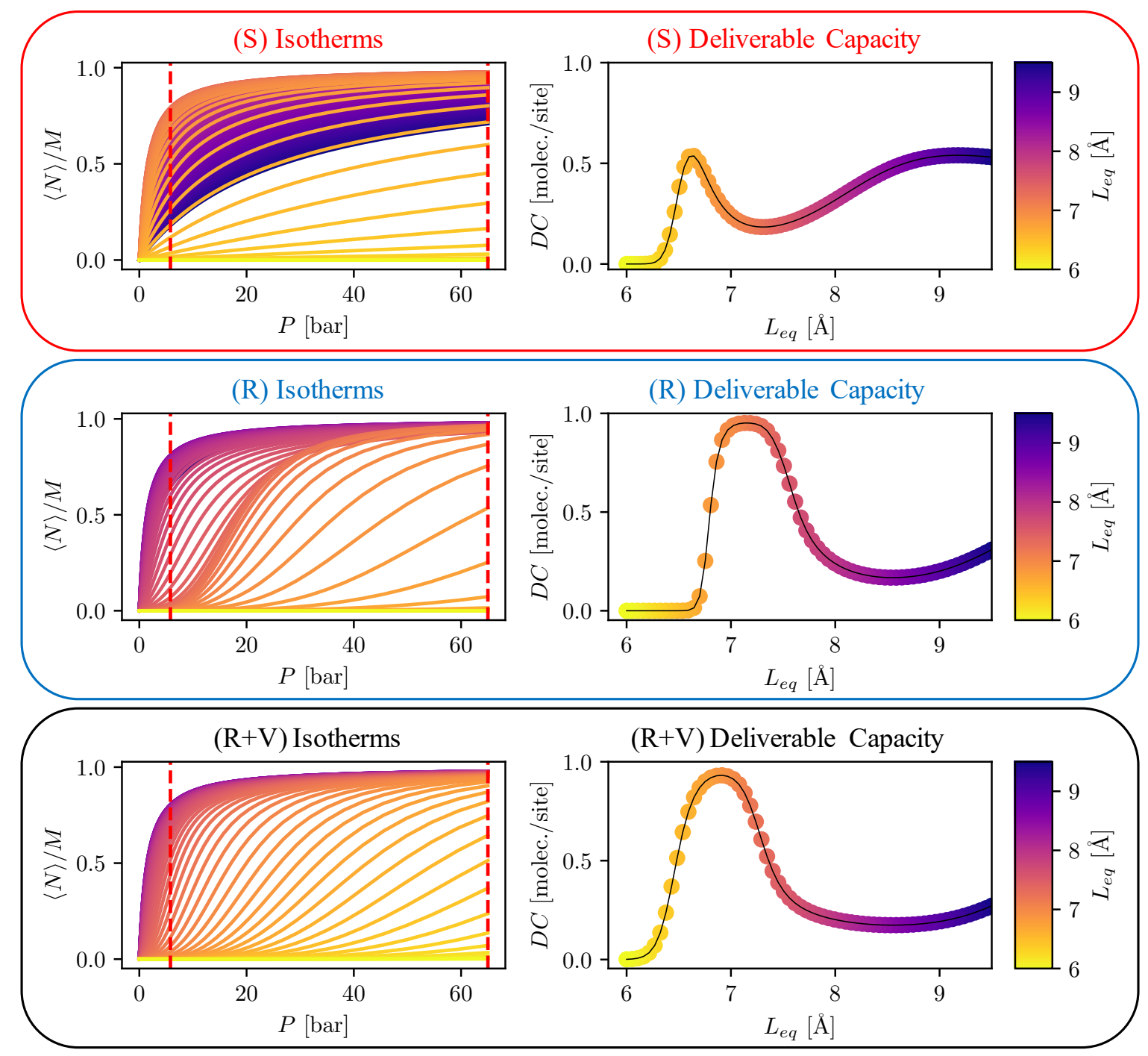

Figure 5: Methane isotherms (color-coded by $L_{e q}$ ) and deliverable capacity plotted as a function of $L_{e q}$ for the static $(\mathrm{S})$, rotating $(\mathrm{R})$, and rotating and vibrating $(\mathrm{R}+\mathrm{V})$ slit pores with $M=4$. For the $(\mathrm{S})$ slit pore, $\theta_{e q}=0$, and for the $(\mathrm{R}+\mathrm{V})$ slit pore, $k=50 \mathrm{~kJ} / \mathrm{mol}$. Dashed red lines indicate $P_{a d s}$ and $P_{d e s}$.

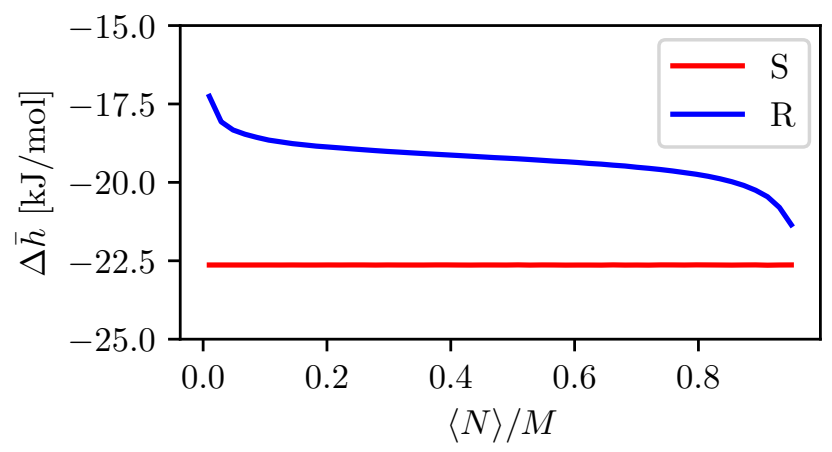

Figure 6: $\Delta \bar{h}$ as a function of loading for the $\mathrm{S}$ and $\mathrm{R}$ slit pore models. in a reduced $\Delta \bar{h}$. This reduced $\Delta \bar{h}$ results in less heat that needs to be added/removed during desorption/adsorption cycling, thereby reducing space, cost, and engineering complexity associated with on-board thermal management systems. We note that our limitation of neglecting adsorbate-adsorbate attraction should yield an underestimation of $\Delta \bar{h}$ at higher loadings, as the adsorption energy would become more favorable when other adsorbates are already present. 


\section{Volumetric deliverable capacity}

The technical targets for gas deliverable capacity are often specified on a volumetric basis, i.e., the original ARPA-E targets required a deliverable capacity of $315 \mathrm{v}(\mathrm{STP}) / \mathrm{v}$. Therefore we convert the model's adsorbate density from a molecules per site basis to a volumetric basis via

$$
\rho_{\text {vol }}=\frac{\langle N\rangle}{M V_{\text {site }} N_{\text {av }}} \frac{R T}{P_{\text {atm }}}[=] \frac{\mathrm{v}(\mathrm{STP})}{\mathrm{v}},
$$

where $P_{a t m}$ and $T$ are standard pressure and temperature and $N_{a v}$ is Avogadro's number. We obtain saturation capacity on a volumetric basis, $\sigma_{v o l}$, if we take $\langle N\rangle / M \rightarrow 1$ in eq. (15), which corresponds to taking $P_{a d s} \rightarrow \infty$. Replotting the volumetric adsorbate densities and volumetric deliverable capacity of the static and rotating slit pores in Figure 7 reveals several important insights.

First, the capacity at the charging pressure of our idealized static slit pore model, $\rho_{v o l}$ at $P_{a d s}$, is consistent with GCMC simulations of methane adsorption in a bilayer graphene structure as a function of separation distance, $L_{e q}$, between each sheet (see S2.2). This indicates that the model's site volume discretization closely reproduces the adsorption of an analogous atomistic simulation. Second, the static pore exhibits an optimal $D C_{v o l} \sim 170$ $\mathrm{v}(\mathrm{STP}) / \mathrm{v}$, and the highest reported deliverable capacities for rigid MOFs are $\sim 200 \mathrm{v}(\mathrm{STP}) / \mathrm{v}$, again validating the adsorption volume discretization. In the case of the top-performing HKUST-1, only $75 \%$ of the 65 bar adsorption capacity, $\rho_{v o l} \sim 270 \mathrm{v}(\mathrm{STP}) / \mathrm{v}$, is converted to deliverable capacity, $D C_{v o l} \sim 200 \mathrm{v}(\mathrm{STP}) / \mathrm{v}$; and these numbers are only further reduced when MOF densification is required. Most importantly, since the rotating slit pore model can translate $\sim 95 \%$ of its adsorption capacity to $D C$ (Figure 5), a porous material that exactly mimics the geometry and potential energy surface of the model could significantly outperform the best known rigid materials.

The caveat is of course that the model has a highly idealized geometry that will be difficult to engineer exactly into a synthesizable
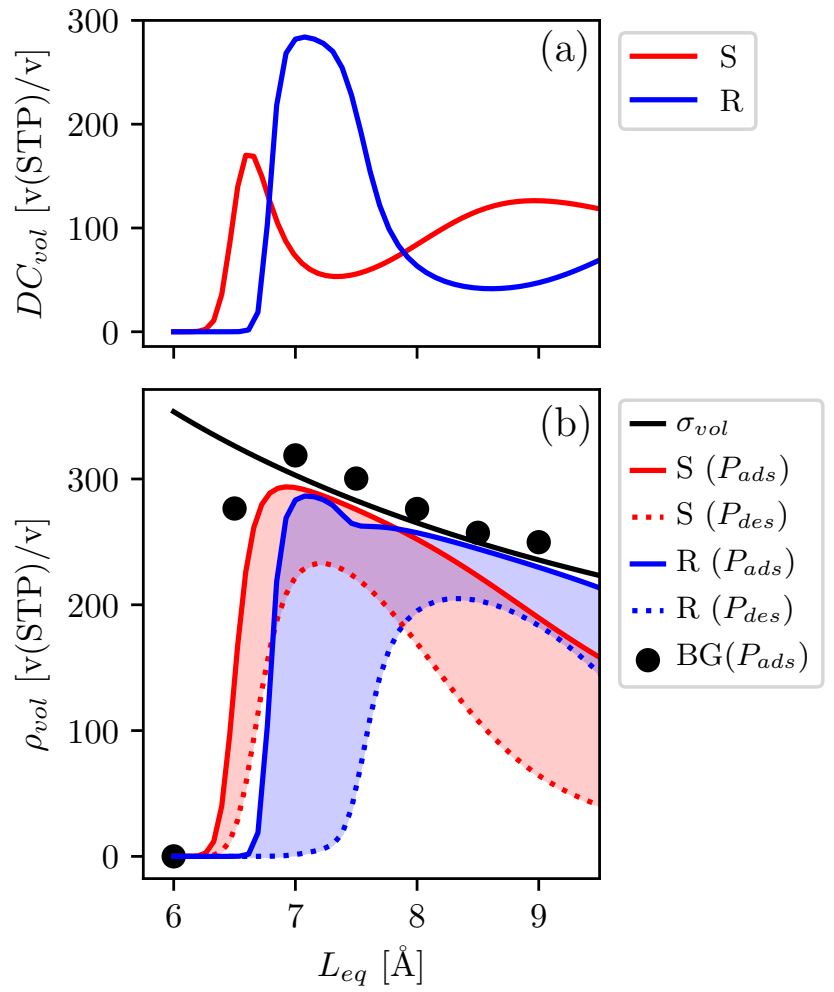

Figure 7: (a) $D C_{v o l}$ for the static $\left(\theta_{e q}=0\right)$ and rotating slit pore as a function of $L_{e q}$. (b) $\rho_{v o l}$ at the adsorption $\left(P_{a d s}=65\right.$ bar $)$, desorption $\left(P_{\text {des }}=5.8 \mathrm{bar}\right)$, and at saturation for both $\mathrm{S}$ and $\mathrm{R}$ as a function of $L_{e q}$. The $\rho_{v o l}$ of bilayer graphene $(\mathrm{BG})$ as predicted by GCMC validates the model's site volume discretization.

crystal structure, and $D C_{v o l}$ is still constrained by the model's upper bound on adsorption capacity of $\sim 290 \mathrm{v}(\mathrm{STP}) / \mathrm{v}$. Finally, we previously noted the limitation of our discretization for large $L$. Figure 7 shows that as $L_{e q}$ starts to increase above $9 \AA$, the $\rho_{v o l}$ for the layered graphene GCMC simulations starts to deviate from $\sigma_{v o l}$, indicating the creation of more adsorption capacity (i.e. more sites per volume) than can be accounted for by our discretization strategy. This range of $L$ for which the discretized model breaks down, however, is well beyond the $6.5<L_{e q}<7.5 \AA$ range of interest for the $\mathrm{R}$ slit pore where the significant $D C$ enhancement occurs. 


\section{Nonporous to porous without vol- ume change in $\mathrm{M}(\mathrm{NDC})$}

The highly idealized geometry of the adsorption model raises the open question of how closely a synthesizable porous material can match it. The recently synthesized ${ }^{29} \mathrm{M}(\mathrm{NDC})$ provides evidence that some of the most important structural/energetic properties of the flexible slit pore model are exhibited in known materials. After removing the $8 \mathrm{DMF}$ solvent molecules (one per open metal site in our simulation cell), DFT relaxation of the empty structure $\left(\left.\mathrm{F}\right|_{\mathrm{F}} ^{\mathrm{DFT}}\right)$ yields a nonporous configuration via rotation of the NDC ligands (see Figure 8). If we instead replace each DMF with a methane molecule, DFT relaxation maintains the same porous state where all slit pores remain aligned in parallel. Deleting the DMF from the experimental structure $\left(\left.\mathrm{F}\right|_{\mathrm{F} \cdot 8 \mathrm{DMF}} ^{\text {Exp. }}\right)$ or deleting methane from the DFT optimized structure $\left(\left.\mathrm{F}\right|_{\mathrm{F} \cdot 8 \mathrm{CH}_{4}} ^{\mathrm{DFT}}\right)$ yields pores that can accommodate methane, while $\left.\mathrm{F}\right|_{\mathrm{F}} ^{\mathrm{DFT}}$ is nonporous. Table 1 shows the $\mathrm{Zeo}++$ computed geometric properties of these configurations where $D_{i}$ is the largest included sphere, $D_{f}$ is the largest free sphere, $V$ is the crystal cell volume, $V_{0}$ is the crystal cell volume of the $\left.\mathrm{F}\right|_{\mathrm{F}} ^{\mathrm{DFT}}$ configuration, and $V_{\text {occ }} / V$ is "probe occupiable" (probe diameter $=3.4 \AA$ ) pore volume $^{61,62}$ fraction that includes both the accessible and non-accessible volumes. Notably, $V / V_{0}$ expansion is practically negligible in comparison to other phase change methane adsorbents, whereas $V_{o c c} / V$ increases drastically. The de-

Table 1: Largest included sphere, $D_{i}[=] \AA$, largest free sphere, $D_{f}[=] \AA$, volume relative to the $\left.\mathrm{F}\right|_{\mathrm{F}} ^{\mathrm{DFT}}$ state, $V / V_{0}$, and occupiable volume fraction, $V_{\text {occ }} / V$, for $\mathrm{M}(\mathrm{NDC})$ porous and nonporous configurations.

\begin{tabular}{|c|c|c|c|c|c|}
\hline & Config. & $D_{i}$ & $D_{f}$ & $V / V_{0}$ & $V_{o c c} / V$ \\
\hline 0 & $\left.\mathrm{~F}\right|_{\mathrm{F} \cdot 8 \mathrm{DMF}} ^{\text {Exp. }}$ & 3.8 & 2.4 & 1.02 & 0.11 \\
\hline 8 & $\left.\mathrm{~F}\right|_{\mathrm{F}} ^{\mathrm{DFT}}$ & 3.4 & 2.4 & 1 & 0 \\
\hline 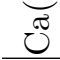 & $\left.\mathrm{F}\right|_{\mathrm{F} \cdot 8 \mathrm{CH}_{4}} ^{\mathrm{DFT}}$ & 3.8 & 2.4 & 1.04 & 0.06 \\
\hline రิ & $\left.\mathrm{F}\right|_{\mathrm{F} \cdot 8 \mathrm{DMF}} ^{\text {Exp. }}$ & 3.9 & 2.6 & 1.00 & 0.23 \\
\hline 是 & $\left.\mathrm{F}\right|_{\mathrm{F}} ^{\mathrm{DFT}}$ & 3.5 & 2.7 & 1 & 0.01 \\
\hline क्र & $\left.\mathrm{F}\right|_{\mathrm{F} \cdot 8 \mathrm{CH}_{4}} ^{\mathrm{DFT}}$ & 4.0 & 2.6 & 1.03 & 0.21 \\
\hline
\end{tabular}

formation pattern between the nonporous and porous states visually mimics the rotating slit pore adsorption model as shown in Figure 8a,b and Figure 8d,e. Figure 8c and Figure 8f show the pore size distributions of the various states.

To gauge whether these materials have favorable methane adsorption properties requires more than the geometric analysis above. Namely, we compute energetics of the adsorbate-driven porous to nonporous transition described by (14) and summarize them in Table 2. Several F.G configurations are considered, with $G \in\left\{1 \mathrm{CH}_{4}, 8 \mathrm{CH}_{4}, 16 \mathrm{CH}_{4}\right\}$. In $1 \mathrm{CH}_{4}$, the structure is optimized with just one methane per simulation box to probe the binding energy and framework deformation energy associated with the first adsorption event. $8 \mathrm{CH}_{4}$ corresponds to one methane at each open metal site, and $16 \mathrm{CH}_{4}$ corresponds to an overall density of two methane molecules per open metal site, i.e. saturation loading. Despite the energy penalty required to deform

Table 2: The average binding energy, $\Delta \bar{E}_{b}$, framework deformation energy, $\Delta \bar{E}_{\text {def }}$, and net energy change, $\Delta \bar{E}_{n e t}[=] \mathrm{kJ} / \mathrm{mol}$ per $\mathrm{CH}_{4}$ adsorbed. Volume relative to the $\left.\mathrm{F}\right|_{\mathrm{F}} ^{\mathrm{DFT}}$ state, $V / V_{0}$, for various $\mathrm{F} \cdot \mathrm{G}$ loadings in $\mathrm{Ca}(\mathrm{NDC})$ and $\mathrm{Sr}(\mathrm{NDC})$.

\begin{tabular}{|c|c|c|c|c|c|}
\hline & $\mathrm{F} \cdot \mathrm{G}$ & $\Delta \bar{E}_{b}$ & $\Delta \bar{E}_{d e f}$ & $\Delta \bar{E}_{n e t}$ & $V / V_{0}$ \\
\hline z & $1 \mathrm{CH}_{4}$ & -22.6 & 14.4 & -8.2 & 1.01 \\
\hline & $8 \mathrm{CH}_{4}$ & -27.3 & 7.4 & -19.9 & 1.04 \\
\hline లే & $16 \mathrm{CH}_{4}$ & -22.2 & 5.3 & -16.9 & 1.05 \\
\hline 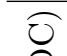 & $1 \mathrm{CH}_{4}$ & -32.8 & 15.2 & -17.6 & 1.02 \\
\hline & $8 \mathrm{CH}_{4}$ & -32 & 3.0 & -29.2 & 1.03 \\
\hline & $16 \mathrm{CH}_{4}$ & -26.1 & 2.1 & -24.0 & 1.03 \\
\hline
\end{tabular}

the framework from the nonporous to porous state, the net adsorption process is favorable for all loadings considered. Importantly, a "collective" behavior is evident Table 2. $\Delta \bar{E}_{\text {def }}$ is large for $\mathrm{G}=1 \mathrm{CH}_{4}$ since it induces a local deformation, i.e., opening of a single slit pore. This energy penalty drops significantly when all slit pores are aligned in the open configuration at $\mathrm{G}=8 \mathrm{CH}_{4}$. Once the slit pores are open, an additional methane can adsorb $\left(\mathrm{G}=16 \mathrm{CH}_{4}\right)$, albeit at slightly lower binding energy per methane. The final result is that 
(a)

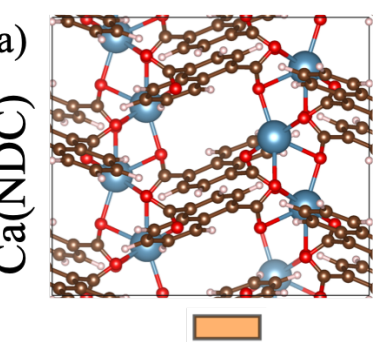

(b)
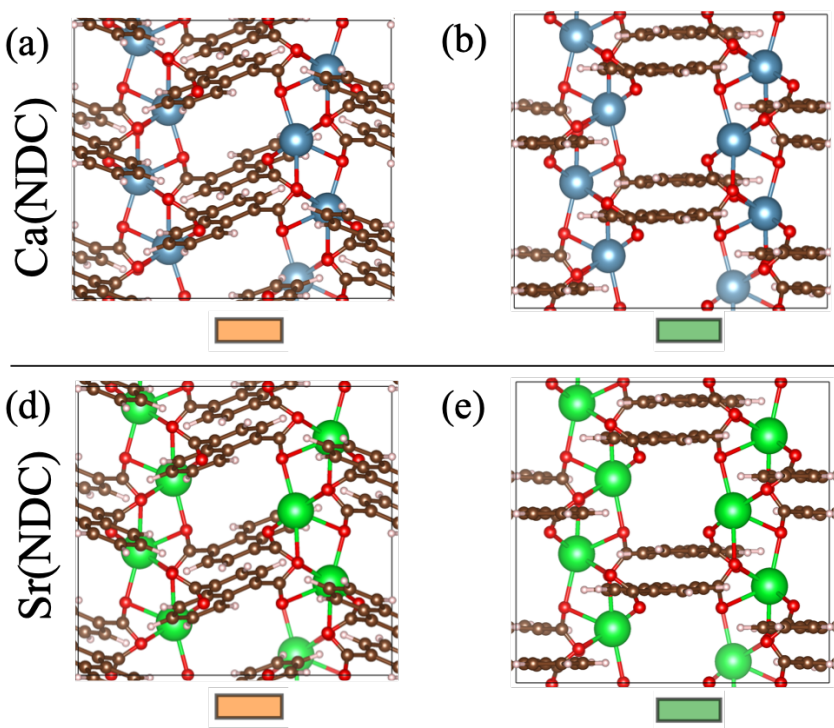

(e)

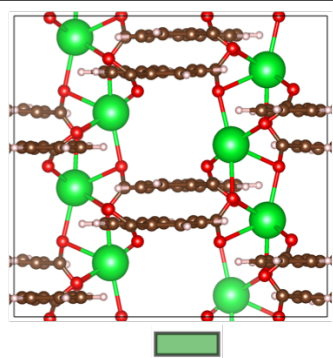

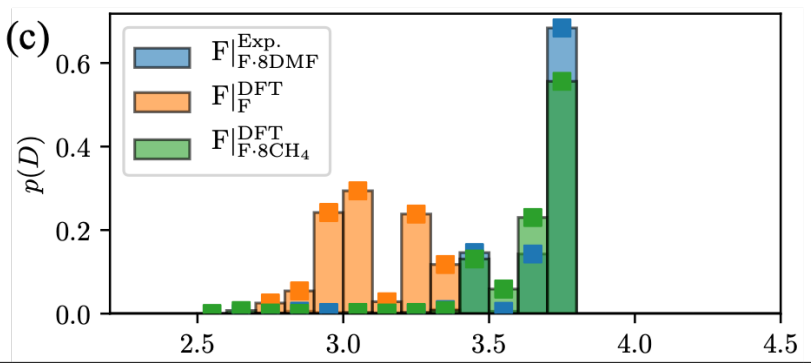

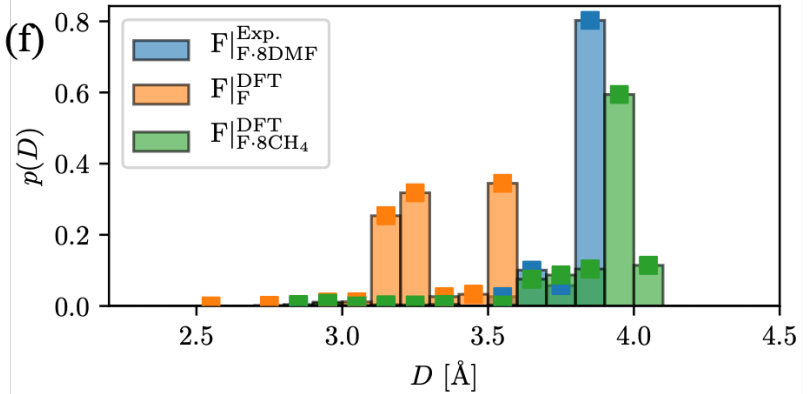

Figure 8: (a,d) show $\left.\mathrm{F}\right|_{\mathrm{F}} ^{\mathrm{DFT}}$ configurations, (b,e) show $\left.\mathrm{F}\right|_{\mathrm{F} \cdot 8 \mathrm{CH}_{4}} ^{\mathrm{DFT}}$ configurations, and (c,f) show the corresponding pore size distribution plots for $\mathrm{Ca}(\mathrm{NDC})$ (a-c) and $\mathrm{Sr}(\mathrm{NDC})(\mathrm{d}-\mathrm{f})$.

the net energy decrease of adsorbing methane is more favorable near saturation than in the empty framework.

The ratio of cell volumes between the porous and nonporous states, $V / V_{o}$, is very close to 1. However, the difference in GCMC predicted isotherms between the $\left.\mathrm{F}\right|_{\mathrm{F}} ^{\mathrm{DFT}}$ and $\left.\mathrm{F}\right|_{\mathrm{F} \cdot 8 \mathrm{CH}_{4}} ^{\mathrm{DFT}}$ configurations in $\mathrm{Sr}$ (NDC) is drastic, as shown in Figure 9, due to the large increase in $V_{o c c} / V$. Force field information and GCMC settings are provided in S2.2. ${ }^{41,42,63}$ How, and at what pressure, any S-shaped adsorption profile occurs will clearly depend on the underlying framework and framework-guest potential energy surface, although we are unaware of any specialized MOF force fields ${ }^{64-67}$ designed specifically for this Sr coordination environment that also capture the non-bonded interactions between methane and the open metal site. ${ }^{68-71}$ Nonetheless, the true isotherm will be bounded between the $\left.\mathrm{F}\right|_{\mathrm{F}} ^{\mathrm{DFT}}$ and $\left.\mathrm{F}\right|_{\mathrm{F} \cdot 8 \mathrm{CH}_{4}} ^{\mathrm{DFT}}$ isotherms. Therefore, the deliverable capacity cannot exceed $\sim 150$ $\mathrm{v}(\mathrm{STP}) / \mathrm{v}$.

\section{Experimental Activation}

$\mathrm{Sr}$ (NDC) was readily synthesized in large quantities (see S3) and good agreement was obtained between the powder x-ray diffraction (PXRD) of the as-synthesized sample and the previously

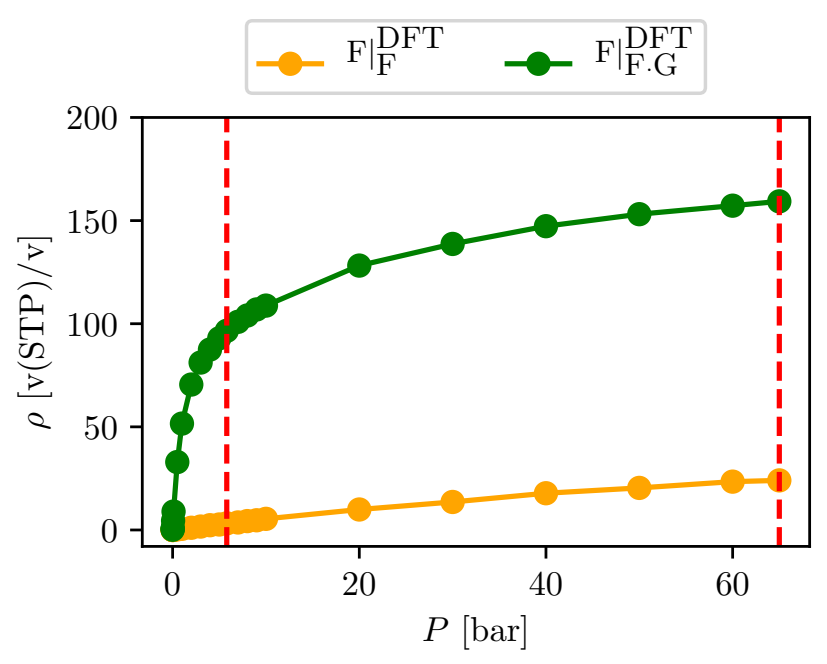

Figure 9: GCMC predicted isotherms for the $\left.\mathrm{F}\right|_{\mathrm{F}} ^{\mathrm{DFT}}$ and $\left.\mathrm{F}\right|_{\mathrm{F} \cdot \mathrm{G}} ^{\mathrm{DFT}}\left(\mathrm{G}=8 \mathrm{CH}_{4}\right)$ configurations of $\mathrm{Sr}$ (NDC). 
reported structure (see Figure 10 and S3 for experimental details). Extreme activation conditions were originally found to be necessary to overcome the strong binding between the DMF solvent and the open metal site and allow it to release through the narrow pore constrictions in the framework, ${ }^{29}$ and we found that heating at $300{ }^{\circ} \mathrm{C}$ for 16 hours led to a structural change in the PXRD pattern of the activated sample. The primary reflection is preserved, suggesting that the unit cell remains unchanged. Nonetheless, no gas uptake was experimentally observed; so while $\operatorname{Sr}(\mathrm{NDC})$ may be an academically interesting manifestation of the slit pore model, more work remains to discover a slit pore material whose $D_{f}$ is not prohibitively small for methane adsorption.

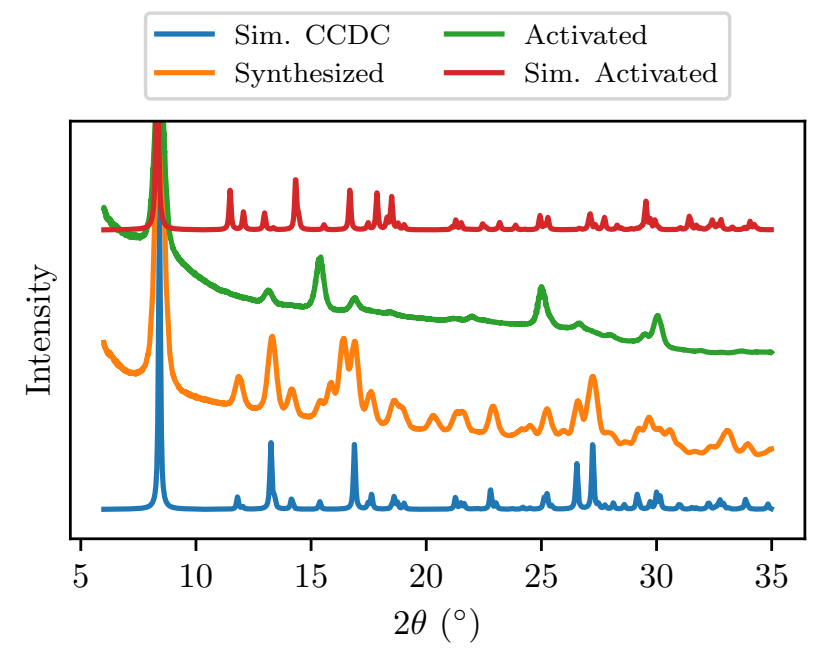

Figure 10: Comparison of the simulated PXRD of the CCDC structure (code KIYMAI), our as-synthesized material, our activated material, and our simulated activated material (corresponding to the $\left.\mathrm{F}\right|_{\mathrm{F}} ^{\mathrm{DFT}}$ structure).

\section{Isoreticular expansion of M(NDC)}

The deficiencies with $\operatorname{Sr}(\mathrm{NDC})$, and where it differs from the adsorption model, are twofold. First, the individual slit pores are staggered slightly, which leads to a $D_{i} \neq D_{f}$. This problematically low $D_{f}$ means that the barrier to methane diffusion will be large and points to kinetics-limited adsorption (note this manifests in the extremely high desorption temperatures, $\sim 300{ }^{\circ} \mathrm{C}$, required to remove $\mathrm{DMF}$ and activate the material). ${ }^{29}$ The second issue is that the probe accessible pore volume of the open structure is not large enough, evidenced by the $\sim 150 \mathrm{v}(\mathrm{STP}) / \mathrm{v}$ saturation capacity of the $\left.\mathrm{F}\right|_{\mathrm{F} \cdot \mathrm{G}} ^{\mathrm{DFT}}$ isotherm in Figure 9. More void volume could be imparted by an isoreticular expansion with a larger planar linker, as long as the energy minimized state in the absence of adsorbates remains nonporous. We performed isoreticular expansion of $\mathrm{Sr}(\mathrm{NDC})$ using hypothetical linkers and a 1D rod MOF assembly algorithm, ${ }^{72}$ then repeated our porosity and adsorption energetics analysis (detailed in S2.3). Even though the isoreticular analogs indeed show the same slit pore rotation, the expanded linkers introduce varying degrees of porosity into the $\left.\mathrm{F}\right|_{\mathrm{F}} ^{\mathrm{DFT}}$ state, which limits $D C$ improvements. Nonetheless, it demonstrates that careful rational design of MOF structures can be useful, and perhaps will even be necessary, to design the ultimate deliverable capacity materials via this flexible slit pore approach.

\section{Discussion and Conclusions}

The results of our intrinsically flexible slit pore model highlight some interesting challenges and opportunities in the continual search for adsorbents with optimal gas deliverable capacity. The deliverable capacities of the rotating slit pore model are, at minimum, a promising indicator that the best gas storage materials may be (as of yet) undiscovered and surpass benchmark rigid and extrinsically flexible adsorbents. This arises from an adsorption induced, nonporous to porous transition that can occur with little to no unit cell volume change. The first challenge in accomplishing this is alluded to in Figure 5. The $L_{e q}$ window over which the optimal rotating slit pore exists is $\sim 0.5 \AA$. In other words, a computational approach to quantitatively screen potential adsorbents must have an extremely accurate potential energy surface. While significant progress has been made in MOF force field development, no single universal force field exists that is highly accurate for all the complex chemistry and topologies exhibited by MOFs. Second, new materials would 
have to be tested or simulated that are nominally nonporous. Fully flexible simulations are required to evaluate their potential, ${ }^{35,36}$ and therefore would have remained undiscovered from rigid screening studies, for which there are very few exceptions. ${ }^{37,38}$ Third, one must find an adsorbent that mimics the idealized flexible slit pore model as closely as possible in order to maximize deliverable capacity.

These challenges notwithstanding, we utilized the insights from the adsorption model to identify a known MOF that displays most, but not all, of the advantages of the slit pore model. Namely, DFT calculations reveal it exhibits a nonporous minimum energy state, and undergoes an adsorbate-driven, energetically favorable, and constant volume transformation to a porous state due to a slit pore rotation. We additionally noted that both $D_{f}$ and $V_{\text {occ }} / V$ are too small and constitute sufficient limitations that $\mathrm{Sr}(\mathrm{NDC})$ cannot achieve the desired methane uptake properties. We sought to address these shortcomings via isoreticular expansion of the framework with a hypothetical linker, which introduced its own complications. Nonetheless, $\operatorname{Sr}(\mathrm{NDC})$ provides clear evidence that the basic concept of the flexible slit pore can be engineered into a porous material. With further refinement and careful rational materials design, a similar material with non-staggered slit pores and a larger probe occupiable pore volume than $\mathrm{Sr}$ (NDC) could provide deliverable capacity that approaches the ideal performance of the slit pore model and exceeds the best known benchmark materials. Concerted and extensive computational efforts will be needed to screen porous materials at the most accurate levels of theory and with fully flexible treatment to uncover them and motivate experimental testing.

Ultimately, this work outlines a less-explored path to discover the ultimate gas deliverable capacity materials via a concrete inverse design objective. Synthetic control over the chemical and structural properties of MOFs is always improving, and the structural motifs that give this adsorption model its outstanding deliverable capacities are increasingly being realized. For example, "adsorbaphores" (large con- jugated linkers arranged in a parallel orientation) ${ }^{73}$ and "nanographene" molecules ${ }^{74}$ have been shown to provide advantageous adsorption properties when incorporated as MOF linkers. Further focus on these types of materials could lead to the discovery of a flexible slit pore geometry that provides the exact deliverable capacity advantages highlighted in this work. Most importantly, this adsorption model provides (1) a template for the rational materials design and (2) thermodynamic justification that the current methane deliverable capacity record holders could be surpassed by materials that undergo little to no volume change. This structural motif is not inherently limited to MOFs and should be explored/sought out in other systems known for gas storage capabilities, such as porous organic cages, covalent organic frameworks, etc.

\section{Conflicts of interest}

There are no conflicts to declare.

\section{Acknowledgments}

The authors gratefully acknowledge research support from the U.S. Department of Energy, Office of Energy Efficiency and Renewable Energy, Hydrogen and Fuel Cell Technologies Office through the Hydrogen Storage Materials Advanced Research Consortium (HyMARC) under Contract Number DE-AC0494AL85000 with Sandia National Laboratories and Contract Number DE-AC02-05CH11231 with Lawrence Berkeley National Laboratory. Sandia National Laboratories is a multimission laboratory managed and operated by National Technology and Engineering Solutions of Sandia, LLC., a wholly owned subsidiary of Honeywell International, Inc., for the U.S. Department of Energy's National Nuclear Security Administration under contract DE-NA-0003525. We acknowledge the use of the Athena supercomputer through the HPC Midlands+ Consortium, and the ARCHER supercomputer through membership of the UK's HPC Materials Chemistry Consortium, which are funded 
by EPSRC Grants No. EP/P020232/1 and No. EP/R029431/1, respectively. We gratefully acknowledge Prof. Jeffrey R. Long for helpful discussions and measurements performed in his laboratory at Lawrence Berkeley National Laboratory.

\section{References}

(1) Menon, V. C.; Komarneni, S. Porous adsorbents for vehicular natural gas storage: a review. J. Porous Mater. 1998, 5, 43-58.

(2) Yang, J.; Sudik, A.; Wolverton, C.; Siegel, D. J. High capacity hydrogenstorage materials: attributes for automotive applications and techniques for materials discovery. Chem. Soc. Rev. 2010, 39, 656675 .

(3) He, Y.; Zhou, W.; Qian, G.; Chen, B. Methane storage in metal-organic frameworks. Chem. Soc. Rev. 2014, 43, 56575678 .

(4) Allendorf, M. D. et al. An assessment of strategies for the development of solidstate adsorbents for vehicular hydrogen storage. Energy Environ. Sci. 2018, 11, 2784-2812.

(5) Goldsmith, J.; Wong-Foy, A. G.; Cafarella, M. J.; Siegel, D. J. Theoretical Limits of Hydrogen Storage in MetalOrganic Frameworks: Opportunities and Trade-Offs. Chem. Mater. 2013, 25, 33733382 .

(6) Gómez-Gualdrón, D. A.; Wilmer, C. E.; Farha, O. K.; Hupp, J. T.; Snurr, R. Q. Exploring the Limits of Methane Storage and Delivery in Nanoporous Materials. $J$. Phys. Chem. C 2014, 118, 6941-6951.

(7) Simon, C. M.; Kim, J.; GomezGualdron, D. A.; Camp, J. S.; Chung, Y. G.; Martin, R. L.; Mercado, R.; Deem, M. W.; Gunter, D.; Haranczyk, M.; Sholl, D. S.; Snurr, R. Q.; Smit, B. The materials genome in action: identifying the performance limits for methane storage. Energy Environ. Sci. 2015, 8, 1190-1199.

(8) Thornton, A. W.; Simon, C. M.; Kim, J.; Kwon, O.; Deeg, K. S.; Konstas, K.; Pas, S. J.; Hill, M. R.; Winkler, D. A.; Haranczyk, M.; Smit, B. Materials Genome in Action: Identifying the Performance Limits of Physical Hydrogen Storage. Chem. Mater. 2017, 29, 2844-2854.

(9) DeCoste, J. B.; Weston, M. H.; Fuller, P. E.; Tovar, T. M.; Peterson, G. W.; LeVan, M. D.; Farha, O. K. Metal-Organic Frameworks for Oxygen Storage. Angew. Chemie Int. Ed. 2014, 53, 14092-14095.

(10) Simon, C. M.; Kim, J.; Lin, L.-C.; Martin, R. L.; Haranczyk, M.; Smit, B. Optimizing nanoporous materials for gas storage. Phys. Chem. Chem. Phys. 2014, 16, 5499 .

(11) Kaija, A. R.; Wilmer, C. E. High-Pressure Methane Adsorption in Porous LennardJones Crystals. J. Phys. Chem. Lett. 2018, 9, 4275-4281.

(12) Kapelewski, M. T.; Runčevski, T.; Tarver, J. D.; Jiang, H. Z. H.; Hurst, K. E.; Parilla, P. A.; Ayala, A.; Gennett, T.; FitzGerald, S. A.; Brown, C. M.; Long, J. R. Record High Hydrogen Storage Capacity in the Metal-Organic Framework $\mathrm{Ni}_{2}$ (m-dobdc) at Near-Ambient Temperatures. Chem. Mater. 2018, 30, 8179-8189.

(13) Kitagawa, S.; Kitaura, R.; Noro, S. I. Functional porous coordination polymers. Angew. Chemie - Int. Ed. 2004, 43, 23342375 .

(14) Férey, G.; Serre, C. Large breathing effects in three-dimensional porous hybrid matter: facts, analyses, rules and consequences. Chem. Soc. Rev. 2009, 38, 1380.

(15) Krause, S.; Hosono, N.; Kitagawa, S. Die Chemie verformbarer poröser Kristalle 
- Strukturdynamik und Gasadsorptionseigenschaften. Angew. Chemie 2020, 132, 15438-15456.

(16) Serre, C.; Millange, F.; Thouvenot, C.; Noguès, M.; Marsolier, G.; Louër, D.; Férey, G. Very large breathing effect in the first nanoporous chromium(III)-based solids: MIL-53 or $\mathrm{Cr}^{\mathrm{III}}(\mathrm{OH}) \cdot\left\{\mathrm{O}_{2} \mathrm{C}_{-} \mathrm{C}_{6} \mathrm{H}_{4}\right.$ $\left.\mathrm{CO}_{2}\right\} \cdot\left\{\mathrm{HO}_{2} \mathrm{C}-\mathrm{C}_{6} \mathrm{H}_{4}-\mathrm{CO}_{2} \mathrm{H}\right\}_{x} \cdot \mathrm{H}_{2} \mathrm{O}_{y} \cdot J$. Am. Chem. Soc. 2002, 124, 13519-13526.

(17) Coudert, F.-X.; Jeffroy, M.; Fuchs, A. H.; Boutin, A.; Mellot-Draznieks, C. Thermodynamics of Guest-Induced Structural Transitions in Hybrid Organic-Inorganic Frameworks. J. Am. Chem. Soc. 2008, 130, 14294-14302.

(18) Bousquet, D.; Coudert, F.-X.; Boutin, A. Free energy landscapes for the thermodynamic understanding of adsorptioninduced deformations and structural transitions in porous materials. J. Chem. Phys. 2012, 137, 044118.

(19) Mason, J. A.; Oktawiec, J.; Taylor, M. K.; Hudson, M. R.; Rodriguez, J.; Bachman, J. E.; Gonzalez, M. I.; Cervellino, A.; Guagliardi, A.; Brown, C. M.; Llewellyn, P. L.; Masciocchi, N.; Long, J. R. Methane storage in flexible metal-organic frameworks with intrinsic thermal management. $\mathrm{Na}$ ture 2015, 527, 357-361.

(20) Keupp, J.; Schmid, R. Molecular Dynamics Simulations of the "Breathing" Phase Transformation of MOF Nanocrystallites. Adv. Theory Simulations 2019, 2, 1900117.

(21) Wang, T. C.; White, J. L.; Bie, B.; Deng, H.; Edgington, J.; Sugar, J. D.; Stavila, V.; Allendorf, M. D. Design Rules for Metal-Organic Framework Stability in High-Pressure Hydrogen Environments. ChemPhysChem 2019, 20, 1305-1310.

(22) Tian, T.; Zeng, Z.; Vulpe, D.; Casco, M. E.; Divitini, G.; Midgley, P. A.; Silvestre-Albero, J.; Tan, J.-C.;
Moghadam, P. Z.; Fairen-Jimenez, D. A sol-gel monolithic metal-organic framework with enhanced methane uptake. Nat. Mater. 2018, 17, 174-179.

(23) Lin, Z.-J.; Lü, J.; Hong, M.; Cao, R. Metal-organic frameworks based on flexible ligands (FL-MOFs): structures and applications. Chem. Soc. Rev. 2014, 43, 5867-5895.

(24) Schneemann, A.; Bon, V.; Schwedler, I.; Senkovska, I.; Kaskel, S.; Fischer, R. A. Flexible metal-organic frameworks. Chem. Soc. Rev. 2014, 43, 6062-6096.

(25) Schneemann, A.; Bloch, E. D.; Henke, S.; Llewellyn, P. L.; Long, J. R.; Fischer, R. A. Influence of Solvent-Like Sidechains on the Adsorption of Light Hydrocarbons in Metal-Organic Frameworks. Chem. - A Eur. J. 2015, 21, 18764-18769.

(26) Kanoo, P.; Matsuda, R.; Sato, H.; Li, L.; Hosono, N.; Kitagawa, S. Pseudo-Gated Adsorption with Negligible Volume Change Evoked by Halogen-Bond Interaction in the Nanospace of MOFs. Chem. - A Eur. J. 2020, 26, 2148-2153.

(27) Evans, J. D.; Krause, S.; Kaskel, S.; Sweatman, M. B.; Sarkisov, L. Exploring the thermodynamic criteria for responsive adsorption processes. Chem. Sci. 2019, 10, 5011-5017.

(28) Li, B.; Wen, H.-M.; Wang, H.; Wu, H.; Tyagi, M.; Yildirim, T.; Zhou, W.; Chen, B. A Porous Metal-Organic Framework with Dynamic Pyrimidine Groups Exhibiting Record High Methane Storage Working Capacity. J. Am. Chem. Soc. 2014, 136, 6207-6210.

(29) Raja, D. S.; Luo, J.-H.; Yeh, C.-T.; Jiang, Y.-C.; Hsu, K.-F.; Lin, C.-H. Novel alkali and alkaline earth metal coordination polymers based on 1,4naphthalenedicarboxylic acid: synthesis, structural characterization and properties. CrystEngComm 2014, 16, 1985. 
(30) Flexibility in a Metal-Organic Framework Material Controlled by Weak Dispersion Forces: The Bistability of MIL-53(Al). Angew. Chemie Int. Ed. 2010, 49, 75017503.

(31) Simon, C. M.; Braun, E.; Carraro, C.; Smit, B. Statistical mechanical model of gas adsorption in porous crystals with dynamic moieties. Proc. Natl. Acad. Sci. 2017, 114, E287-E296.

(32) Elsaidi, S. K.; Mohamed, M. H.; Simon, C. M.; Braun, E.; Pham, T.; Forrest, K. A.; Xu, W.; Banerjee, D.; Space, B.; Zaworotko, M. J.; Thallapally, P. K. Effect of ring rotation upon gas adsorption in SIFSIX-3-M ( $\mathrm{M}=\mathrm{Fe}$, $\mathrm{Ni})$ pillared square grid networks. Chem. Sci. 2017, 8, 2373-2380.

(33) Coudert, F. X. The osmotic framework adsorbed solution theory: Predicting mixture coadsorption in flexible nanoporous materials. Phys. Chem. Chem. Phys. 2010, 12, 10904-10913.

(34) Zang, J.; Nair, S.; Sholl, D. S. Osmotic ensemble methods for predicting adsorption-induced structural transitions in nanoporous materials using molecular simulations. J. Chem. Phys. 2011, 134, 184103.

(35) Witman, M.; Wright, B.; Smit, B. Simulating Enhanced Methane Deliverable Capacity of Guest Responsive Pores in Intrinsically Flexible MOFs. J. Phys. Chem. Lett. 2019, 5929-5934.

(36) Rogge, S. M. J.; Goeminne, R.; Demuynck, R.; Gutiérrez-Sevillano, J. J.; Vandenbrande, S.; Vanduyfhuys, L.; Waroquier, M.; Verstraelen, T.; Van Speybroeck, V. Modeling Gas Adsorption in Flexible Metal-Organic Frameworks via Hybrid Monte Carlo/Molecular Dynamics Schemes. Adv. Theory Simulations 2019, 2, 1800177.

(37) Witman, M.; Ling, S.; Jawahery, S.; Boyd, P. G.; Haranczyk, M.; Slater, B.;
Smit, B. The Influence of Intrinsic Framework Flexibility on Adsorption in Nanoporous Materials. J. Am. Chem. Soc. 2017, 139, 5547-5557.

(38) Agrawal, M.; Sholl, D. S. Effects of Intrinsic Flexibility on Adsorption Properties of Metal-Organic Frameworks at Dilute and Nondilute Loadings. ACS Appl. Mater. Interfaces 2019, 11, 31060-31068.

(39) Yaghi, O. M.; O'Keeffe, M.; Ockwig, N. W.; Chae, H. K.; Eddaoudi, M.; Kim, J. Reticular synthesis and the design of new materials. Nature 2003, 423, 705-714.

(40) Deng, H.; Grunder, S.; Cordova, K. E.; Valente, C.; Furukawa, H.; Hmadeh, M.; Gandara, F.; Whalley, A. C.; Liu, Z.; Asahina, S.; Kazumori, H.; O'Keeffe, M.; Terasaki, O.; Stoddart, J. F.; Yaghi, O. M. Large-Pore Apertures in a Series of MetalOrganic Frameworks. Science 2012, 336, 1018-1023.

(41) Chen, B.; Siepmann, J. I. Transferable Potentials for Phase Equilibria. 3. ExplicitHydrogen Description of Normal Alkanes. J. Phys. Chem. B 1999, 103, 5370-5379.

(42) Rappe, A. K.; Casewit, C. J.; Colwell, K. S.; Goddard, W. A.; Skiff, W. M. UFF, a full periodic table force field for molecular mechanics and molecular dynamics simulations. J. Am. Chem. Soc. 1992, 114, 10024-10035.

(43) Zhao, X. A simple function for calculating the interaction between a molecule and a graphene sheet. arXiv: 2012, 1209.4248.

(44) Ghysels, A.; Vanduyfhuys, L.; Vandichel, M.; Waroquier, M.; Van Speybroeck, V.; Smit, B. On the Thermodynamics of Framework Breathing: A Free Energy Model for Gas Adsorption in MIL-53. J. Phys. Chem. C 2013, 117, 11540-11554. 
(45) Jelic, V.; Marsiglio, F. The double-well potential in quantum mechanics: A simple, numerically exact formulation. Eur. J. Phys. 2012, 33, 1651-1666.

(46) Frenkel, D.; Smit, B. Understanding Molecular Simulations; Elsevier: San Diego, 2002.

(47) Torres-Knoop, A.; Poursaeidesfahani, A.; Vlugt, T. J. H.; Dubbeldam, D. Behavior of the Enthalpy of Adsorption in Nanoporous Materials Close to Saturation Conditions. J. Chem. Theory Comput. 2017, 13, 3326-3339.

(48) Myers, A. L. Chem. Thermodyn. Ind.; 2004; pp 243-252.

(49) VandeVondele, J.; Krack, M.; Mohamed, F.; Parrinello, M.; Chassaing, T.; Hutter, J. Quickstep: Fast and accurate density functional calculations using a mixed Gaussian and plane waves approach. Comput. Phys. Commun. 2005, 16\%, 103-128.

(50) Hutter, J.; Iannuzzi, M.; Schiffmann, F.; VandeVondele, J. cp2k: atomistic simulations of condensed matter systems. Wiley Interdiscip. Rev. Comput. Mol. Sci. 2014, 4, 15-25.

(51) VandeVondele, J.; Hutter, J. Gaussian basis sets for accurate calculations on molecular systems in gas and condensed phases. J. Chem. Phys. 2007, 127, 114105.

(52) Goedecker, S.; Teter, M.; Hutter, J. Separable dual-space Gaussian pseudopotentials. Phys. Rev. B 1996, 54, 1703-1710.

(53) Krack, M. Pseudopotentials for $\mathrm{H}$ to $\mathrm{Kr}$ optimized for gradient-corrected exchange-correlation functionals. Theor. Chem. Acc. 2005, 114, 145-152.

(54) Perdew, J. P.; Burke, K.; Ernzerhof, M. Generalized Gradient Approximation Made Simple. Phys. Rev. Lett. 1996, 7ry, 3865-3868.
(55) Grimme, S.; Antony, J.; Ehrlich, S.; Krieg, H. A consistent and accurate ab initio parametrization of density functional dispersion correction (DFT-D) for the 94 elements H-Pu. J. Chem. Phys. 2010, 132, 154104 .

(56) Liu, L.; Chen, Z.; Wang, J.; Zhang, D.; Zhu, Y.; Ling, S.; Huang, K.-W.; Belmabkhout, Y.; Adil, K.; Zhang, Y.; Slater, B.; Eddaoudi, M.; Han, Y. Imaging defects and their evolution in a metalorganic framework at sub-unit-cell resolution. Nature Chemistry 2019, 11, 622628.

(57) Ling, S.; Slater, B. Dynamic acidity in defective UiO-66. Chem. Sci. 2016, 7, 47064712 .

(58) Nanthamathee, C.; Ling, S.; Slater, B.; Attfield, M. P. Contradistinct Thermoresponsive Behavior of Isostructural MIL53 Type Metal-Organic Frameworks by Modifying the Framework Inorganic Anion. Chem. Mater. 2015, 27, 85-95.

(59) Boys, S.; Bernardi, F. The calculation of small molecular interactions by the differences of separate total energies. Some procedures with reduced errors. Mol. Phys. 1970, 19, 553-566.

(60) Tong, M.; Lan, Y.; Qin, Z.; Zhong, C. Computation-Ready, Experimental Covalent Organic Framework for Methane Delivery: Screening and Material Design. $J$. Phys. Chem. C 2018, 122, 13009-13016.

(61) Willems, T. F.; Rycroft, C. H.; Kazi, M.; Meza, J. C.; Haranczyk, M. Algorithms and tools for high-throughput geometrybased analysis of crystalline porous materials. Microporous Mesoporous Mater. 2012, 149, 134-141.

(62) Ongari, D.; Boyd, P. G.; Barthel, S.; Witman, M.; Haranczyk, M.; Smit, B. Accurate Characterization of the Pore Volume in Microporous Crystalline Materials. Langmuir 2017, 33, 14529-14538. 
(63) Dubbeldam, D.; Calero, S.; Ellis, D. E.; Snurr, R. Q. RASPA: molecular simulation software for adsorption and diffusion in flexible nanoporous materials. Mol. Simul. 2016, 42, 81-101.

(64) Tafipolsky, M.; Amirjalayer, S.; Schmid, R. First-Principles-Derived Force Field for Copper Paddle-WheelBased Metal-Organic Frameworks. $J$. Phys. Chem. C 2010, 114, 14402-14409.

(65) Bristow, J. K.; Tiana, D.; Walsh, A. Transferable Force Field for MetalOrganic Frameworks from FirstPrinciples: BTW-FF. J. Chem. Theory Comput. 2014, 10, 4644-4652.

(66) Addicoat, M. A.; Vankova, N.; Akter, I. F.; Heine, T. Extension of the Universal Force Field to Metal-Organic Frameworks. J. Chem. Theory Comput. 2014, 10, 880-891.

(67) Jawahery, S.; Rampal, N.; Moosavi, S. M.; Witman, M.; Smit, B. Ab Initio Flexible Force Field for Metal-Organic Frameworks Using Dummy Model Coordination Bonds. J. Chem. Theory Comput. 2019, 15, 3666-3677.

(68) Dzubak, A. L.; Lin, L.-C.; Kim, J.; Swisher, J. A.; Poloni, R.; Maximoff, S. N.; Smit, B.; Gagliardi, L. Ab initio carbon capture in open-site metalorganic frameworks. Nat. Chem. 2012, 4, 810-816.

(69) Lin, L. C.; Lee, K.; Gagliardi, L.; Neaton, J. B.; Smit, B. Force-field development from electronic structure calcula- tions with periodic boundary conditions: Applications to gaseous adsorption and transport in metal-organic frameworks. $J$. Chem. Theory Comput. 2014, 10, $1477-$ 1488 .

(70) Koh, H. S.; Rana, M. K.; Wong-Foy, A. G.; Siegel, D. J. Predicting Methane Storage in Open-Metal-Site Metal-Organic Frameworks. J. Phys. Chem. C 2015, 119, 13451-13458.

(71) Mercado, R.; Vlaisavljevich, B.; Lin, L.-C.; Lee, K.; Lee, Y.; Mason, J. A.; Xiao, D. J.; Gonzalez, M. I.; Kapelewski, M. T.; Neaton, J. B.; Smit, B. Force Field Development from Periodic Density Functional Theory Calculations for Gas Separation Applications Using Metal-Organic Frameworks. J. Phys. Chem. C 2016, 120, 12590-12604.

(72) Witman, M.; Ling, S.; Anderson, S.; Tong, L.; Stylianou, K. C.; Slater, B.; Smit, B.; Haranczyk, M. In silico design and screening of hypothetical MOF74 analogs and their experimental synthesis. Chem. Sci. 2016, 7, 6263-6272.

(73) Boyd, P. G. et al. Data-driven design of metal-organic frameworks for wet flue gas CO2 capture. Nature 2019, 576, 253-256.

(74) Suginome, S.; Sato, H.; Hori, A.; Mishima, A.; Harada, Y.; Kusaka, S.; Matsuda, R.; Pirillo, J.; Hijikata, Y.; Aida, T. One-Step Synthesis of an Adaptive Nanographene MOF: Adsorbed GasDependent Geometrical Diversity. J. Am. Chem. Soc. 2019, 141, 15649-15655. 


\section{Graphical TOC Entry}

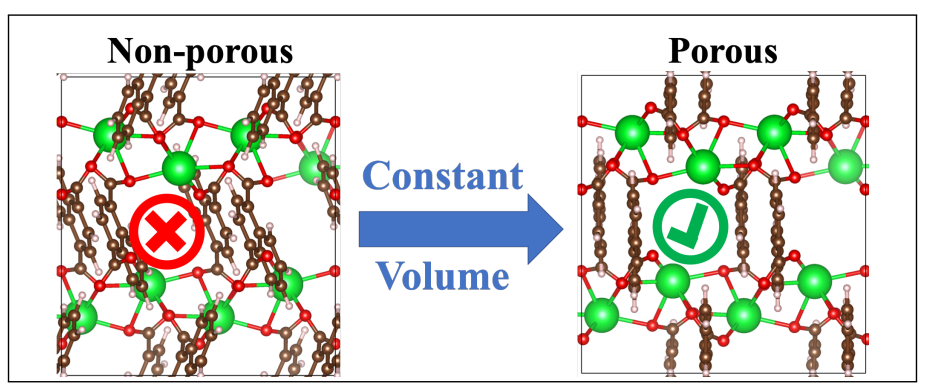

